\title{
PUSHING DOWN INFINITE LOEB MEASURES
}

\author{
DAVID A. ROSS
}

\begin{abstract}
Sufficient conditions are given under which the standard part map on an arbitrary Hausdorff space can be used to push down an infinite nonstandard measure. This makes it easier to construct standard infinite Borel measures using nonstandard techniques.
\end{abstract}

\section{Introduction}

A common way nonstandard analysis is used to construct a Borel measure on a topological space $X$ is to first construct a Loeb measure $\mu_{L}$ on a companion space $\Omega$ (usually a subset of ${ }^{*} X$ ), and then 'push' $\mu_{L}$ down to $X$ using the standard part map st. This requires that st be measurable from the (complete) Loeb $\sigma$-algebra on $\Omega$ to the Borel $\sigma$-algebra on $X$. The question of when st is measurable has been extensively studied; see [4] and [9] for early papers on the question, and the discussion following Theorem 3.2 of [16] for more recent results. The reader is also referred to [8], [2], [11], [12], as well as to [5] and [6] for a functional approach.

Infinite measures have traditionally been especially problematic. Reasons include greater difficulty in proving that st is measurable, and difficulty in keeping mass in $\mu_{L}$ from escaping to infinity. For example, in the recent paper [10], had the authors attempted to simply push the measure down with the standard part with respect to the product topology, then every nonempty set would have received infinite measure. On the other hand, the standard part map with respect to the box topology would in some cases have assigned zero measure to any compact set. As a result, some results which would have been natural in the setting of an infinite measure have been stated and proved just for finite measures. Examples include the author's results [14] and [15].

This paper adapts a technique from [10] to prove a pushing down result for infinite Loeb measures. To illustrate how this theorem can be used in practice, the proof from [15] is revisited and generalized to construct a (possibly infinite) measure on a space which need not be compact.

Received September 11, 2006; in revised form April 23, 2007. 
The author is grateful to the University of Illinois for its hospitality, and to the referee for useful comments and suggestions.

\section{Preliminaries}

Assume that the nonstandard model is as saturated as it needs to be to carry out all constructions; in particular, it is $\kappa$-saturated for some $\kappa$ larger than the cardinality of any standard set that we might encounter.

$(\Omega, \mathscr{A}, \mu)$ will always denote an internal, *finitely-additive *measure space. We recall the existence of the Loeb completion of ${ }^{\circ} \mu$ (see [16] or [18]). ${ }^{\circ} \mu$ is a standard, extended-real-valued finitely-additive measure on the algebra $\mathscr{A}$, even if $\mu(\Omega)$ is externally infinite. Moreover, by saturation ${ }^{\circ} \mu$ is automatically countably additive on $\mathscr{A}$, so the machinery of the usual Carathéodory construction now applies. In particular, ${ }^{\circ} \mu$ extends to a measure on a $\sigma$-algebra containing $\mathscr{A}$ (see [17] Chapter 12, Theorem 8). Note that the construction holds for externally infinite measures, though it will only be applied in this paper to subsets of $\Omega$ with externally finite measure. Some of the technical issues that arise when starting with an externally infinite measure are detailed in [18].

Lemma 1. There is a complete measure space $\left(\Omega, \mathscr{A}_{L}, \mu_{L}\right)$ such that:

(i) $\mathscr{A} \subseteq \mathscr{A}_{L}$;

(ii) For every $A \in \mathscr{A}, \mu_{L}(A)={ }^{\circ} \mu(A)$ (where by convention ${ }^{\circ} \mu(A)=\infty$ if $\mu(A)$ is infinite);

(iii) For every $E \in \mathscr{A}_{L}$ with $\mu_{L}(E)$ finite, $\mu_{L}(E)=\sup \left\{{ }^{\circ} \mu(A): A \subseteq\right.$ $E, A \in \mathscr{A}\}$

The next lemma, for the special case of $A_{i} \in \mathscr{A}$, was proved in [7] using an idea from [4].

Lemma 2. Let $\left\{A_{i}\right\}_{i \in I}$ be a family of internal sets such that each $A_{i} \in \mathscr{A}_{L}$ and $\operatorname{card}(I)<\kappa$. Suppose ${ }^{\circ} \mu(\Omega)<\infty$. Then $\bigcup_{i} A_{i} \in \mathscr{A}_{L}$ and $\bigcap_{i} A_{i} \in \mathscr{A}_{L}$.

Proof. It suffices to prove that $\bigcap_{i} A_{i} \in \mathscr{A}_{L}$. We may suppose that $\left\{A_{i}\right\}_{i \in I}$ is closed under finite intersection. Let $r=\inf _{i} \mu_{L}\left(A_{i}\right)$, let $A_{i_{n}}, n \in \mathrm{N}$ be a decreasing sequence with $\lim _{n} \mu\left(A_{i_{n}}\right)=r$, and put $A_{\infty}=\bigcap_{n} A_{i_{n}}$.

Let $s<r$, and $i_{1}, i_{2}, \ldots, i_{n} \in I$. For some $j \in I, A_{j}=A_{i_{1}} \cap \cdots \cap A_{i_{n}}$, and $\mu_{L}\left(A_{j}\right) \geq r>s$. By Lemma 1 there is a $B_{s} \in \mathscr{A}$ with $B_{s} \subseteq A_{j}$ and $\mu_{L}\left(B_{s}\right)>$ $s$. By $\kappa$-saturation there is a $B_{s} \in \mathscr{A}$ for which $B_{s} \subseteq A_{i}$ for every $i \in I$, and $\mu_{L}\left(B_{s}\right)>s$. Put $B_{\infty}=\bigcup_{s \in \mathrm{Q}, s<r} B_{s}$. Then $B_{\infty} \subseteq \bigcap_{i} A_{i} \subseteq \bigcap_{n} A_{i_{n}}=A_{\infty}$, and $\mu_{L}\left(B_{\infty}\right)=r=\mu_{L}\left(A_{\infty}\right)$. 
Let $X$ be a Hausdorff space, $\mathscr{K}=\mathscr{K}_{X}=\{K \subseteq X: K$ compact $\}, \mathscr{B}_{X}$ be the Borel $\sigma$-algebra on $X$, and $N S(X)$ the set of nearstandard elements of * $X$. The following is a straightforward exercise.

Lemma 3. If $K$ is locally compact then $N S(X)=\bigcup_{K \in \mathscr{K}}{ }^{*} K$

The first theorem is mainly contained in already-known results. For example, see the remarks following Proposition 3.4.7 of [1], and the discussion following Theorem 3.2 of [16].

Theorem 1. Suppose $X$ is a locally compact Hausdorff space, $\Omega \subseteq{ }^{*} X$, ${ }^{\circ} \mu \Omega<\infty$, and ${ }^{*} E \cap \Omega \in \mathscr{A}_{L}$ for every open $E \subseteq X$. Then the restriction $\mathrm{st}_{\Omega}$ of st to $\Omega \cap N S(X)$ is $\mathscr{A}_{L}-\mathscr{B}_{X}$ measurable.

Note (as a consequence of this theorem ) that (i) $\mu_{L}$ can be pushed down to a measure $m=\mu_{L} \circ \mathrm{st}_{\Omega}^{-1}$ on $\left(X, \mathscr{B}_{X}\right)$; (ii) $\Omega \cap N S(X) \in \mathscr{A}_{L}$; and (iii) $\mu_{L} \Omega=$ $m(X)$ precisely when $\mu_{L}(\Omega \backslash N S(X))=0$. By (3) of Lemma 1, $\mu_{L}$ is innerregular with respect to the internal sets. (Recall that a measure $v$ is innerregular with respect to a family $\mathscr{F}$ provided that for every measurable set $E$ with $v(E)<\infty, v(E)=\sup \{v(F): F \subseteq E, F \in \mathscr{F}\}$.) For locally-compact $X$ the image of an internal subset of $N S(X)$ under the standard part map is compact; it follows that the measure $m$ constructed in this way will be Radon (that is, inner-regular with respect to compact sets).

Proof. If $K \in \mathscr{K}_{X}$ then ${ }^{*} K \cap \Omega \in \mathscr{A}_{L}$ by hypothesis, so by Lemma 2 ,

$$
\Omega \cap N S(X)=\bigcup\left\{\Omega \cap{ }^{*} K: K \in \mathscr{K}_{X}\right\} \in \mathscr{A}_{L}
$$

Let $C \subseteq X$ be closed. Since $\bigcap\left\{\Omega \cap{ }^{*} U: C \subseteq U \subseteq X, U\right.$ open $\} \in \mathscr{A}_{L}$, it suffices to show that $\operatorname{st}_{\Omega}^{-1} C=\Omega \cap N S(X) \cap \bigcap\left\{{ }^{*} U: C \subseteq U \subseteq X, U\right.$ open $\}$.

If ${ }^{\circ} \omega \in C$ and $U$ is an open superset of $C$ then $\omega \in{ }^{*} U$; this proves the inclusion $\subseteq$. For $\supseteq$, if ${ }^{\circ} \omega=x \notin C$ then for some open neighborhood $v$ of $x$ with compact closure $\bar{v}, \bar{v} \cap C=\emptyset$. Then $\omega \in{ }^{*} v$ but $\omega \notin{ }^{*} \bar{v}^{\complement}$, proving $\supseteq$.

The following is the main result of the paper.

Theorem 2. Suppose $X$ is a Hausdorff space, $\Omega \subseteq{ }^{*} X,{ }^{*} E \cap \Omega \in \mathscr{A}_{L}$ for every open $E \subseteq X$, and for every $K \in \mathscr{K}_{X}, \mu_{L}\left({ }^{*} K \cap \Omega\right)<\infty$. Then for every $K \in \mathscr{K}_{X}, m_{K}(E):=\mu_{L}\left({ }^{*} K \cap \mathrm{st}_{\Omega}^{-1} E\right)$ defines a Borel measure on $X$. Moreover, $m(E):=\sup _{K \in \mathscr{H}_{X}} m_{K}(E)$ defines a Borel measure on $X$, and for every $K \in \mathscr{K}_{X}, m$ and $m_{K}$ agree on the interior of $K$.

Note that there is no requirement here that $\mu(\Omega)$ be finite. As in Theorem 1, the measures $m_{K}$ and $m$ will be Radon. 
Proof. For any $K \in \mathscr{K}_{X}, K$ is a compact Hausdorff space, so by the previous theorem the restriction st ${ } K \cap \Omega$ is measurable from ${ }^{*} K \cap \Omega$ to $K$. If $E \in \mathscr{B}_{X}$ then $\mathrm{st}^{-1}{ }_{K \cap \Omega} E=\mathrm{st}^{-1}{ }_{K \cap \Omega}(E \cap K)$, so $m_{K}$ defines a Borel measure on all of $X$.

Evidently for any $E \in \mathscr{B}_{X}, m_{K} E$ is monotone in $K$.

To see that $m$ is a measure, let $\left\{A_{n}\right\}_{n=1}^{\infty}$ be a sequence of pairwise disjoint Borel subsets of $X$, and put $A=\bigcup_{n=1}^{\infty} A_{i}$. It suffices to show that $m A=$ $\sum_{n} m A_{n}$. One direction is easy: if $K \in \mathscr{K}_{X}$ then $m_{K} A=\sum_{n} m_{K} A_{n} \leq$ $\sum_{n} m A_{n}$. Since $K$ was arbitrary, $m A \leq \sum_{n} m A_{n}$. (In particular, if $m A=\infty$ then $m A=\infty=\sum_{n} m A_{n}$.)

For the opposite inequality, fix $N \in \mathrm{N}^{+}$, let $K_{1}, \ldots, K_{N} \in \mathscr{K}_{X}$, and put $K=K_{1} \cup \cdots \cup K_{N}$. Then

$$
\sum_{n=1}^{N} m_{K_{n}} A_{n} \leq \sum_{n=1}^{N} m_{K} A_{n}=m_{K}\left(\bigcup_{n=1}^{N} A_{n}\right) \leq m A
$$

Since $K_{1}, \ldots, K_{N}$ were arbitrary, $\sum_{n=1}^{N} m A_{n} \leq m A$, so $\sum_{n=1}^{\infty} m A_{n} \leq m A$.

Finally, let $K, M \in \mathscr{K}_{X}$, and $E \subseteq U$ where $U$ is the interior of $K$. Note

$$
\begin{aligned}
{ }^{*} M \cap \mathrm{st}_{\Omega}^{-1} E & ={ }^{*} M \cap \mathrm{st}_{\Omega}^{-1}(E \cap U) \\
& ={ }^{*} M \cap \mathrm{st}_{\Omega}^{-1}(E) \cap \mathrm{st}_{\Omega}^{-1}(U) \\
& \subseteq{ }^{*} M \cap \mathrm{st}_{\Omega}^{-1}(E) \cap{ }^{*} U \\
& \subseteq{ }^{*} M \cap \mathrm{st}_{\Omega}^{-1}(E) \cap{ }^{*} K \\
& \subseteq \mathrm{st}_{\Omega}^{-1}(E) \cap{ }^{*} K
\end{aligned}
$$

so

$$
m_{M} E \leq m_{K} E \leq m E .
$$

Taking the supremum over all $M \in \mathscr{K}_{X}$, we obtain

$$
m E \leq m_{K} E \leq m E,
$$

as desired. 


\section{Applications}

\subsection{Riesz Representation Theorem}

The utility of Theorem 2 is illustrated in this section by employing it to extend the nonstandard proof of the Riesz Representation Theorem from [15] to spaces which are not necessarily compact.

Let $X$ be a locally compact Hausdorff space, and $C_{c}(X)$ be the vector space of continuous real functions on $X$ with compact support. Let $T: C_{c}(X) \rightarrow \mathrm{R}$ be a positive linear functional.

THeOREM 3. There is a Radon measure $m$ on $X$ such that $\int f d m=T f$ for every $f \in C_{c}(X)$

Proof. Write $\mathscr{C}=C_{c}(X)$. Put $\operatorname{Ker}^{+}(T, \mathscr{C})=\{f \in \mathscr{C}: f \geq 0, T f=0\}$. First, consider the case where $\operatorname{Ker}^{+}(T, \mathscr{C})=\{0\}$. (The author notes that this is the only case that was considered in [15].) For $A \subseteq X$ and $f \in \mathscr{C}$ write $f \mid A$ for the restriction of $f$ to $A$.

Let $\mathscr{D} \subseteq \mathscr{C}$ be a basis for $\mathscr{C}$ (as a vector space over R). By saturation there is a hyperfinite $\Omega=\left\{\omega_{1}, \ldots, \omega_{H}\right\} \subseteq{ }^{*} X$ with $x \in \Omega$ for every standard $x \in X$. Let $\left\{f_{1}, \ldots, f_{k}\right\}$ be an arbitrary finite subset of $\mathscr{D}$.

Consider the internal system of linear equations in $\mathbf{p} \in{ }^{*} \mathbf{R}^{H}$ :

$$
\left[\begin{array}{cccc}
* f_{1}\left(\omega_{1}\right) & { }^{*} f_{1}\left(\omega_{2}\right) & \ldots & { }^{*} f_{1}\left(\omega_{H}\right) \\
{ }^{*} f_{2}\left(\omega_{1}\right) & * f_{2}\left(\omega_{2}\right) & \ldots & *{ }_{2}\left(\omega_{H}\right) \\
\vdots & \vdots & & \vdots \\
* f_{k}\left(\omega_{1}\right) & * f_{k}\left(\omega_{2}\right) & \ldots & *{ }_{k}\left(\omega_{H}\right)
\end{array}\right]\left[\begin{array}{c}
p_{1} \\
p_{2} \\
\vdots \\
p_{H}
\end{array}\right]=\left[\begin{array}{c}
T f_{1} \\
T f_{2} \\
\vdots \\
T f_{k}
\end{array}\right]
$$

By the Farkas Lemma from linear algebra [13], there are two mutually exclusive alternatives:

(a) For some $\alpha_{1}, \alpha_{2}, \ldots, \alpha_{k} \in{ }^{*} \mathrm{R}$,

$$
* \sum_{i} \alpha_{i}\left({ }^{*} f_{i} \mid \Omega\right) \geq 0 \quad \text { but } \quad * \sum_{i} \alpha_{i}\left(T f_{i}\right)<0 .
$$

Without loss of generality $\max _{i}\left\{\left|\alpha_{i}\right|\right\}=1$. If $x \in X$ is standard, then $x \in \Omega$, so

$$
\sum_{i}\left({ }^{\circ} \alpha_{i}\right) f_{i}(x) \approx \sum_{i} \alpha_{i}^{*} f_{i}(x) \geq 0,
$$

so $\sum_{i}\left({ }^{\circ} \alpha_{i}\right) f_{i}(x) \geq 0$. By positivity of $T$,

$$
0 \leq T\left(\sum_{i}\left({ }^{\circ} \alpha_{i}\right) f_{i}\right)=\sum_{i}\left({ }^{\circ} \alpha_{i}\right) T f_{i} \approx * \sum_{i} \alpha_{i}\left(T f_{i}\right)<0 ;
$$


it follows that $\sum_{i}\left({ }^{\circ} \alpha_{i}\right) f_{i} \in \operatorname{Ker}^{+}(T, \mathscr{C})=\{0\}$, contradicting linear independence of $\mathscr{D}$.

Therefore, the following alternative must hold.

(b) There is a solution $\mathbf{p} \in{ }^{*} \mathrm{R}^{H}$ of the system with $p_{i} \geq 0,1 \leq i \leq H$.

By saturation in the cardinality of $\mathscr{D}$, there must be such a $\mathbf{p}$ such that for every $f \in \mathscr{D}$, hence every $f \in \mathscr{C}, T f={ }^{*} \sum_{i} p_{i}{ }^{*} f\left(\omega_{i}\right)$. Define an internal measure $\mu$ on $(\Omega, \mathscr{P}(\Omega))$ by putting $\mu\left(\left\{\omega_{i}\right\}\right)=p_{i}$. Note that $\mathscr{P}(\Omega)$ contains every set of the form ${ }^{*} E \cap \Omega$ for standard $E$. If $K \in \mathscr{K}_{X}$, then by local compactness of $X$ there is an $f \in \mathscr{C}, f \geq 0$, such that $f \geq 1$ on $K$, so

$$
\begin{aligned}
\mu\left({ }^{*} K \cap \Omega\right) & =\sum_{\omega \in \in^{*} K \cap \Omega} 1 \mu(\{\omega\}) \leq \sum_{\omega \in \in^{*} K \cap \Omega} f(\omega) \mu(\{\omega\}) \\
& \leq \sum_{\omega \in \Omega} f(\omega) \mu(\{\omega\})=T f<\infty
\end{aligned}
$$

This verifies the conditions of Theorem 2. Let $m$ and $m_{K}\left(K \in \mathscr{K}_{X}\right)$ be the Radon measures on $X$ given in the conclusion of that theorem.

If $f \in \mathscr{C}$, let $U$ be an open set containing the support of $f$ such that $U$ has compact closure $K$. Then

$$
\begin{aligned}
& \int f d m=\int f d m_{K} \quad \text { (since } m=m_{K} \text { on the support of } f \text { ) } \\
& =\int_{{ }^{*} \cap \Omega}{ }^{{ }^{*}} f d \mu_{L} \quad \begin{array}{l}
\left(\text { since } f\left({ }^{\circ} \omega\right)={ }^{*} f(\omega) \text { on }{ }^{*} K\right. \text { and } \\
\text { st }^{*} K \cap \Omega
\end{array} \\
& \approx \int_{{ }^{*} K \cap \Omega}{ }^{*} f d \mu \quad \text { (since } f \text { is bounded) } \\
& =\int_{\Omega}^{*} f d \mu=T f
\end{aligned}
$$

This proves Theorem 3 in the case that $\operatorname{Ker}^{+}(T, \mathscr{C})=\{0\}$. For the more general case, observe first that the proof does not require that $\mathscr{C}$ be all of $C_{c}(X)$, only a linear subspace over $\mathrm{R}$ with the the property that if $K \subseteq X$ is compact then there is a nonnegative $f \in \mathscr{C}$ with $f>0$ on $K$.

Put

$$
U=\left\{x \in X: f(x)>0 \text { for some } f \in \operatorname{Ker}^{+}\left(T, C_{c}(X)\right)\right\}
$$

It is easy to see that $U$ is open. Put $X^{\prime}=X \backslash U$ and $\mathscr{C}^{\prime}=\left\{f \mid X^{\prime}: f \in C_{c}(X)\right\}$.

Proposition 1. If $f, g \in C_{c}(X)$ and $f=g$ on $X^{\prime}$ then $T f=T g$.

Assuming this proposition is true, we may define a positive linear functional $T^{\prime}$ on $\mathscr{C}^{\prime}$ by $T^{\prime}\left(f \mid X^{\prime}\right)=T(f)$. Note that $\operatorname{Ker}^{+}\left(T^{\prime}, \mathscr{C}^{\prime}\right)=\{0\}$, so by the 
previous case of the theorem there is a measure $\mu^{\prime}$ on $X^{\prime}$ with $T^{\prime}\left(f \mid X^{\prime}\right)=$ $\int_{X^{\prime}} f \mid X^{\prime} d \mu^{\prime}$ for $f \in C_{c}(X)$. Extend $\mu^{\prime}$ to $\mu$ on $X$ by $\mu E=\mu^{\prime}\left(E \cap X^{\prime}\right)$, and observe that for $f \in C_{c}(X), T f=T^{\prime}\left(f \mid X^{\prime}\right)=\int_{X^{\prime}} f \mid X^{\prime} d \mu^{\prime}=\int f d \mu$, as desired

It remains to prove Proposition 1. By considering $|f-g|$ it suffices to show that if $f \geq 0$ on $X$ and $f=0$ on $X^{\prime}$ then $T f=0$. Let $K$ be a compact set containing the support of $f$, and let $\gamma$ be a nonnegative function in $C_{c}(X)$ which is strictly positive on $K$. By saturation there is a $\phi \in{ }^{*} \operatorname{Ker}^{+}\left(T, C_{c}(X)\right)$ such that $\phi \geq{ }^{*} h$ for every $h \in \operatorname{Ker}^{+}\left(T, C_{c}(X)\right)$. It is easy to verify that for any $\delta \in \mathrm{R}^{+},{ }^{*} f \leq \phi+\delta^{*} \gamma$. Then $T(f) \leq{ }^{*} T(\phi)+{ }^{*} T\left(\delta^{*} \gamma\right)=\delta T(\gamma)$. Since $\delta$ is arbitrary, $T(f)=0$, proving the proposition and completing Theorem 3 .

\subsection{A Theorem of Choquet}

This argument extends to a variant of Theorem 3 due to Choquet. (The author thanks the referee for suggesting this extension.) If $f, g$ are nonnegative continuous functions on $X$, say that $g$ dominates $f$ if for every $\epsilon>0$ there is some $h \in C_{c}(X)$ with $f \leq \epsilon g+h$ on $X$. Note that this $h$ may always be chosen so that $0 \leq h \leq f$. (To clarify the meaning of domination, the reader is invited to show that $g$ dominates $f$ if and only if for every $x \in{ }^{*} X$ which is not nearstandard, either ${ }^{*} f(x)=0$ or $\frac{{ }^{*} g(x)}{{ }^{*} f(x)}$ is infinite.) Let $\mathscr{H}$ be a linear space of continuous functions on $X$, and let $\mathscr{H}^{+}$be the nonnegative elements of $\mathscr{H}$. Call $\mathscr{H}$ adapted provided

(i) $\mathscr{H}=\mathscr{H}^{+}-\mathscr{H}^{+}$;

(ii) For every $x \in X$ there is an $f \in \mathscr{H}^{+}$with $f(x)>0$; and

(iii) For every $f \in \mathscr{H}^{+}$there is a $g \in \mathscr{H}^{+}$which dominates $f$.

Choquet has proved the following ([3], Theorem 34.6):

THeOREM 4. Let $X$ be a locally compact Hausdorff space, $\mathscr{H}$ an adapted space of continuous functions on $X$, and $T$ a positive linear functional on $\mathscr{H}$. There is then a Radon measure $m$ on $X$ such that every $f \in \mathscr{H}$ is integrable with $\int f d m=T f$

Assume first that $\operatorname{Ker}^{+}(T, \mathscr{H})=\{0\}$.

As in the proof of Theorem 3, there is a hyperfinite $\Omega=\left\{\omega_{1}, \ldots, \omega_{H}\right\} \subseteq{ }^{*} X$ and a nonnegative $\mathbf{p} \in{ }^{*} \mathrm{R}^{H}$ such that for every $f \in \mathscr{H}, T f={ }^{*} \sum_{i} p_{i}{ }^{*} f\left(\omega_{i}\right)$. Let $\mathscr{H}^{\#}$ be the set of continuous functions $f$ with ${ }^{*} \sum_{i} p_{i}{ }^{*} f\left(\omega_{i}\right)$ finite, and for such $f$ put $T^{\#} f={ }^{\circ *} \sum_{i} p_{i}{ }^{*} f\left(\omega_{i}\right)$. Evidently $\mathscr{H} \subseteq \mathscr{H}^{\#}$, and $T^{\#}$ is a positive linear functional on $\mathscr{H}^{\#}$.

Suppose $f \in C_{c}(X)$, and $K$ is a compact set containing the support of $f$. By property (ii) of 'adapted', compactness of $K$, and linearity of $\mathscr{H}$, there is an 
element $g$ of $\mathscr{H}^{+}$with $g(x)>0$ on $K$. We may choose $g$ so that the minimum of $g$ on $K$ is greater than the maximum of $|f|$ on $K$. Then $\left|{ }^{*} \sum_{i} p_{i}{ }^{*} f\left(\omega_{i}\right)\right| \leq$ $\left.{ }^{*} \sum_{i} p_{i}\right|^{*} f\left(\omega_{i}\right) \mid \leq{ }^{*} \sum_{i} p_{i}{ }^{*} g\left(\omega_{i}\right)=T g<\infty$; it follows that $C_{c}(X) \subseteq \mathscr{H}^{\#}$.

By Theorem 3, there is a Radon measure $m$ on $X$ such that $\int f d m=T f$ for every $f \in C_{c}(X)$. It remains to verify this equality more generally for $f \in \mathscr{H}$. Since $\mathscr{H}=\mathscr{H}^{+}-\mathscr{H}^{+}$, it suffices to consider $f \in \mathscr{H}^{+}$.

Since $m$ is Radon, $\int f d m=\sup \left\{\int g d m: 0 \leq g \leq f, g \in C_{c}(X)\right\}=$ $\sup \left\{T g: 0 \leq g \leq f, g \in C_{c}(X)\right\} \leq T f$. For the opposite inequality, let $g \in \mathscr{H}^{+}$dominate $f$, let $\epsilon>0$, and $h \in C_{c}(X)$ with $f \leq \epsilon g+h$ and $0 \leq h \leq f$ on $X$. Then $T f \leq T(\epsilon g+h)=\epsilon T g+T h=\epsilon T g+\int h d m \leq \epsilon T g+\int f d m$. Since $T g$ is finite and $\epsilon$ is arbitrary, $T f \leq \int f d m$.

This proves Theorem 3 in the case that $\operatorname{Ker}^{+}(T, \mathscr{H})=\{0\}$. For the more general case, put $U=\left\{x \in X: f(x)>0\right.$ for some $\left.f \in \operatorname{Ker}^{+}(T, \mathscr{H})\right\}$, $X^{\prime}=X \backslash U$, and $\mathscr{C}^{\prime}=\left\{f \mid X^{\prime}: f \in \mathscr{H}\right\}$. As in the proof of Theorem 3, it suffices to show that if $f \in \mathscr{H}$ and $f=0$ on $X^{\prime}$ then $T f=0$. Let $f^{+} \in \mathscr{H}^{+}$ (respectively, $f^{-} \in \mathscr{H}^{-}$) with $f=f^{+}-f^{-}$. Let $g^{+} \in \mathscr{H}^{+}$(respectively, $\left.g^{-} \in \mathscr{H}^{+}\right)$dominate $f^{+}\left(f^{-}\right)$, let $\epsilon>0$, and find nonnegative $h^{+}, h^{-} \in C_{c}(X)$ with $f^{+} \leq \epsilon g^{+}+h^{+}$and $f^{-} \leq \epsilon g^{-}+h^{-}$. Put $h=h^{+}+h^{-} \in C_{c}(X)$, $g=g^{+}+g^{-} \in \mathscr{H}^{+}$, then $|f|=f^{+}+f^{-} \leq \epsilon g+h$, and we may assume $h \leq|f|$.

Let $K$ be a compact set containing the support of $h$; by the hypothesis that $\mathscr{H}$ is adapted, there is an element $\gamma$ of $\mathscr{H}^{+}$which is strictly positive on $K$. By saturation there is a $\phi \in{ }^{*} \operatorname{Ker}^{+}(T, \mathscr{H})$ such that $\phi \geq{ }^{*} \psi$ for every $\psi \in \mathscr{H}^{+}$. It is easy to verify that ${ }^{*} h \leq \phi+\epsilon^{*} \gamma$, so $T(h) \leq{ }^{*} T(\phi)+{ }^{*} T\left(\epsilon^{*} \gamma\right)=\epsilon T(\gamma)$.

Now, $T(f) \leq T(\epsilon g+h)=\epsilon T(g)+T(h) \leq \epsilon T(g)+\epsilon T(\gamma)$. Since $\epsilon$ is arbitrary, $T(f)=0$.

\section{REFERENCES}

1. Albeverio, Sergio, Høegh-Krohn, Raphael, Fenstad, Jens Erik, and Lindstrøm, Tom, Nonstandard Methods in Stochastic Analysis and Mathematical Physics, Pure Appl. Math. 122 (1986).

2. Aldaz, Jesus, and Render, Hermann, Borel measure extensions of measures defined on sub$\sigma$-algebras, Adv. Math. 150 (2000), no. 2, 233-263.

3. Choquet, Gustave, Lectures on analysis. Vol. II: Representation theory, Edited by J. Marsden, T. Lance and S. Gelbart, W. A. Benjamin, Inc., New York-Amsterdam, 1969.

4. Henson, C. Ward, Analytic sets, Baire sets and the standard part map, Canad. J. Math. 31 (1979), no. 3, 663-672.

5. Landers, D., and Rogge, L., Lower and upper Loeb-integrals, Trans. Amer. Math. Soc. 358 (2006), no. 8, 3263-3283 (electronic).

6. Landers, D., and Rogge, L., On the representation of $\tau$-continuous and Radon functionals with lower and upper Loeb integrals, Internat. J. Pure Appl. Math. 26 (2006), no. 1, 1-20.

7. Landers, Dieter, and Rogge, Lothar, Universal Loeb-measurability of sets and of the standard part map with applications, Trans. Amer. Math. Soc. 304 (1987), no. 1, 229-243. 
8. Lindstrøm, Tom, Pushing down Loeb measures, Preprint Series, Department of Mathematics, University of Oslo, 1981.

9. Loeb, Peter A., Weak limits of measures and the standard part map, Proc. Amer. Math. Soc. 77 (1979), no. 1, 128-135.

10. Loeb, Peter A., and Ross, David A., Infinite products of infinite measures, Illinois J. Math. 49 (2005), no. 1, 153-158 (electronic).

11. Render, Hermann, Pushing down Loeb measures, Math. Scand. 72 (1993), no. 1, 61-84.

12. Render, Hermann, Countably determined sets and a conjecture of $C$. W. Henson, Math. Ann. 303 (1995), no. 2, 307-324.

13. Rockafellar, R. Tyrrell, Convex Analysis, Princeton Math. Ser. 28 (1970).

14. Ross, David A., Measures invariant under local homeomorphisms, Proc. Amer. Math. Soc. 102 (1988), no. 4, 901-905.

15. Ross, David A., Yet another short proof of the Riesz representation theorem, Math. Proc. Cambridge Philos. Soc. 105 (1989), no. 1, 139-140.

16. Ross, David A., Loeb measure and probability, Nonstandard analysis (Edinburgh, 1996), NATO Adv. Sci. Inst. Ser. C Math. Phys. Sci. 493 (1997), 91-120.

17. Royden, H. L., Real Analysis, third ed., Macmillan Publishing Company, New York, 1988.

18. Stroyan, K. D., and Bayod, José Manuel, Foundations of Infinitesimal Stochastic Analysis, Stud. Logic Found. Math. 119 (1986).

DEPARTMENT OF MATHEMATICS

UNIVERSITY OF HAWAII

HONOLULU, HI 96822

USA

E-mail: ross@math.hawaii.edu

URL: www.math.hawaii.edu/ross 